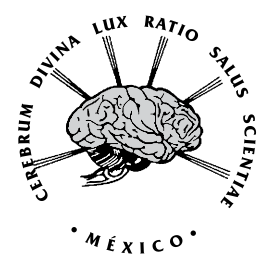

\title{
Determinantes sociales en salud colectiva en mujeres con síndrome metabólico en el medio rural
}

\author{
Social determinants the health collective in women's \\ with metabolic syndrome
}

\author{
Abigail Fernández-Sánchez, ${ }^{*}$ Ma. Guadalupe Ojeda-Vargas, ${ }^{* *}$ Luis Pérez-Álvarez, ${ }^{* * *}$ \\ Ricardo Magos-Núñez ${ }^{* * *}$
}

* Maestra en Ciencias en Enfermería. Profesora Investigadora de tiempo completo de la Universidad Autónoma del Estado de Morelos.

** Doctora en Enfermería. Rectora del Campus Celaya Salvatierra, Universidad de Guanajuato.

*** Doctor en Psicología. Profesor Investigador de tiempo completo de la Universidad Autónoma del Estado de Morelos.

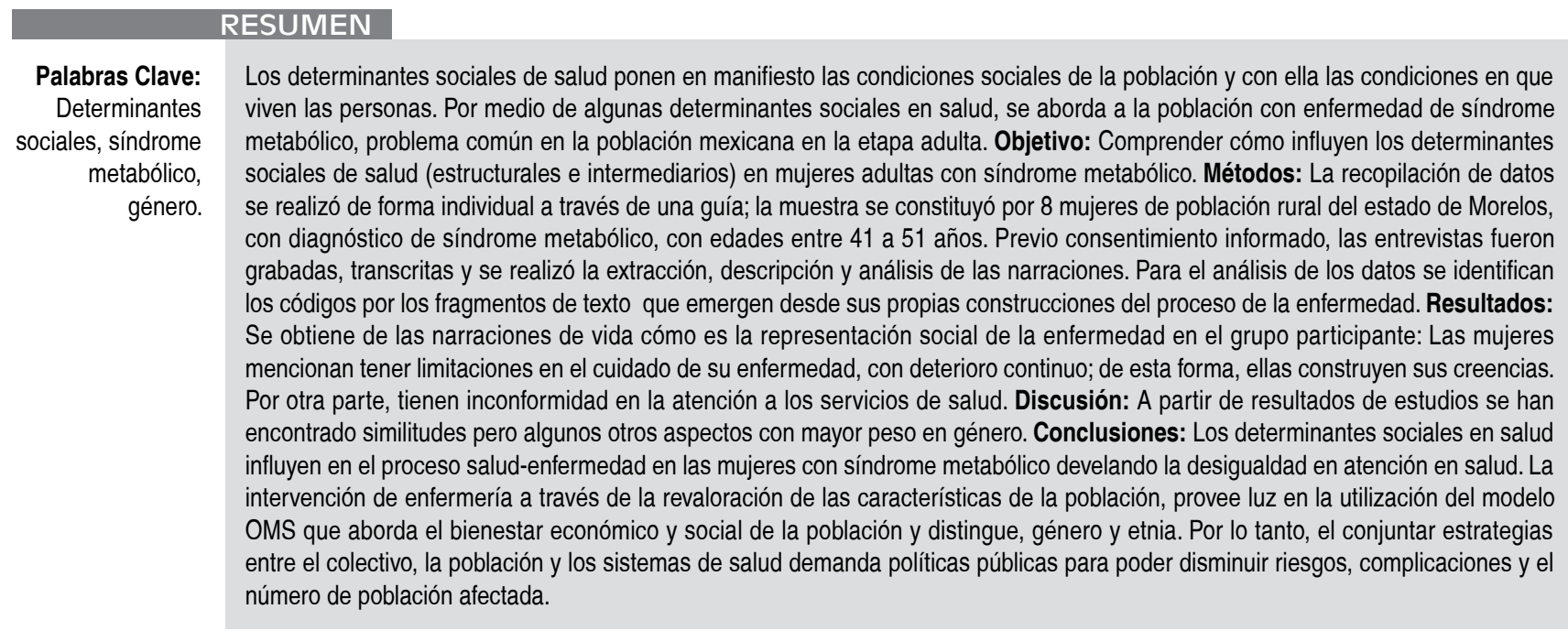

\section{ABSTRACT}

Key words: $\quad$ Social determinants of health manifest social conditions of the population and with it the conditions of the people as they Social live. Through some of the social determinants of health is addressed to people with disease of metabolic syndrome common determinants, metabolic syndrome, problem in the Mexican population from adulthood. Objective: Understand how social determinants influence health (structural and intermediaries) in adult women with the metabolic syndrome. Methods: Data collection was performed individually by a guide; the sample was constituted by 8 women of rural population of the state of Morelos, diagnosed with metabolic syndrome, gende. age between 41-51 years. Informed consent, the interviews were recorded, transcribed, extraction description and analysis of the narratives is performed. For analysis of the data identified by the code snippets emerging from its own constructions of the

Recibido para publicación: 17/04/2014. Aceptado: 17/07/2014. 
disease process, the results are described. Results: They result from narratives of life, such as social representation of the disease in the participant group: mention have limitations in the care of their disease, leading continuous deterioration, thus women construct their beliefs. Moreover they have disagreement on attention to health services. Discussion: From results of studies has found some similarities but other aspects more heavily on gender. Conclusions: The social determinants of health influence the health-disease process in women with metabolic syndrome revealing inequality in health care. Nursing intervention through reprising characteristics of the population provides light when using the WHO model to address the welfare of the economic, social, population, gender, ethnicity (...). Therefore combine collective strategies among the population and health systems, public policies in order to reduce risks complications and the number of people affected.

\section{INTRODUCCIÓN}

Los sistemas de salud y las comunidades científicas han explorado desde hace más de dos décadas los determinantes sociales en salud, puesto que ésta no sólo depende de los servicios médicos ni de las características biológicas humanas, sino también de las condiciones sociales de las personas, cómo viven y trabajan -llamados estilos o modos de vida-, lo cual impacta positiva o negativamente en su salud, y las condiciones sociales o vías particulares presentes en la vida diaria. ${ }^{1,2}$

El sistema de salud en México clama por la salud colectiva, igualdad de oportunidades, mayor libertad, impulso a la competitividad y prevención de riesgos a la salud. No obstante, ocupar el primer lugar a nivel mundial en obesidad infantil y tener las tasas más altas en diabetes y síndrome metabólico, conduce a voltear la mirada ante esta situación. ${ }^{3}$

Los determinantes sociales en la salud. El modelo de la Comisión de los Determinantes Sociales en Salud (CDSS) de la Organización Mundial de la Salud (OMS) parte de un esquema general sobre la determinación social en la enfermedad (Figura 1), ${ }^{1-4}$ sus construcciones en determinantes estructurales que producen inequidades en salud y el bienestar a través de su acción sobre los determinantes intermediarios. Se manifiestan en prácticas individuales, las características del ambiente y la demografía de la población para poder garantizar la satisfacción de las necesidades básicas. ${ }^{5,6}$

El enfoque del modelo de la $\mathrm{CDSS}^{7}$ es de importancia para lograr la transformación propuesta por el sistema de salud, así como la promoción de la cultura y valores sociales, para acortar la brecha entre lo epistemológico y teórico del modelo y los determinantes del cambio social en la población que sufre síndrome metabólico.

El síndrome metabólico (SM) se define como "El conjunto de alteraciones metabólicas constituido por obesidad de distribución central, la disminución del colesterol unido a las lipoproteínas de alta densidad (cHDL), la elevación de triglicéridos, glucosa, y de la presión arterial", ${ }^{10}$ su presencia no sólo se relaciona con una causa bioquímica, también los determinantes sociales (como sedentarismo, estilos de vida, la alimentación, la pobreza, entre otros) conllevan a su aparición. ${ }^{10}$

EI SM se ha situado como un problema de salud pública del siglo XXI, lo que ha modificado la morbimortalidad tanto a nivel mundial como nacional, llevando a una elevada prevalencia en la sociedad, y con tendencia al aumento. Lo anterior afecta la calidad de vida de la población, así como la falta de tratamientos necesarios en el primer nivel de atención, por los escasos presupuestos sanitarios. ${ }^{9}$

Por otra parte, están los años de vida saludable perdidos por causa del SM, el incremento de la mortalidad prematura y discapacidad y la mayor prevalencia de este síndrome entre las mujeres. De aquí surge el interés de realizar este estudio para conocer cómo influyen los determinantes sociales en salud en mujeres con SM desde la aparición del mismo, en una población rural.

\section{MÉTODO}

Se realizó un estudio de tipo cualitativo; participaron mujeres adultas de una población rural del estado de Morelos. El muestreo fue por saturación teórica. El estudio fue por narrativa de vida, Minayo, ${ }^{11}$ para investigar el sentido de la experiencia humana común en lugares sociales específicos y apreciar una versión posible, lo que atribuyen, cómo vivenciaron los hechos a partir de sus propios datos, su experiencia, su conocimiento y su visión del futuro.

En la selección de las participantes se consideró que cubrieran los criterios de heterogeneidad; género: mujeres con SM, edad: entre 42 y 51 años.

La recolección de la información se realizó a través de una entrevista semiestructurada, elaborada por los investigadores. Se llevó a cabo una entrevista en profundidad en el domicilio de las participantes, previa cita, la aplicación fue de manera individual. Se solicitó el consentimiento informado, en el cual se le comunicaba el propósito de la investigación y la confidencialidad. Conforme a las consideraciones éticas de la Declaración de Helsinki, de la AMM y de la Ley General de Salud en el Artículo $3^{\circ}$, "La investigación para la salud", apartados I y III, así como la Comisión de Ética de la Secretaría de Salud, conforme al reglamento de la Ley General de Salud en materia de investigación para la salud. ${ }^{12}$

Por medio de una Guía de Entrevista se recabaron los datos sociodemográficos, cuya categoría central son los determinantes sociales en salud. Las entrevistas fueron grabadas y transcritas literalmente; se obtuvieron 56 códigos y subcategorías (Cuadro I) de forma ordenada desde el mayor a menor impacto, para posteriormente 


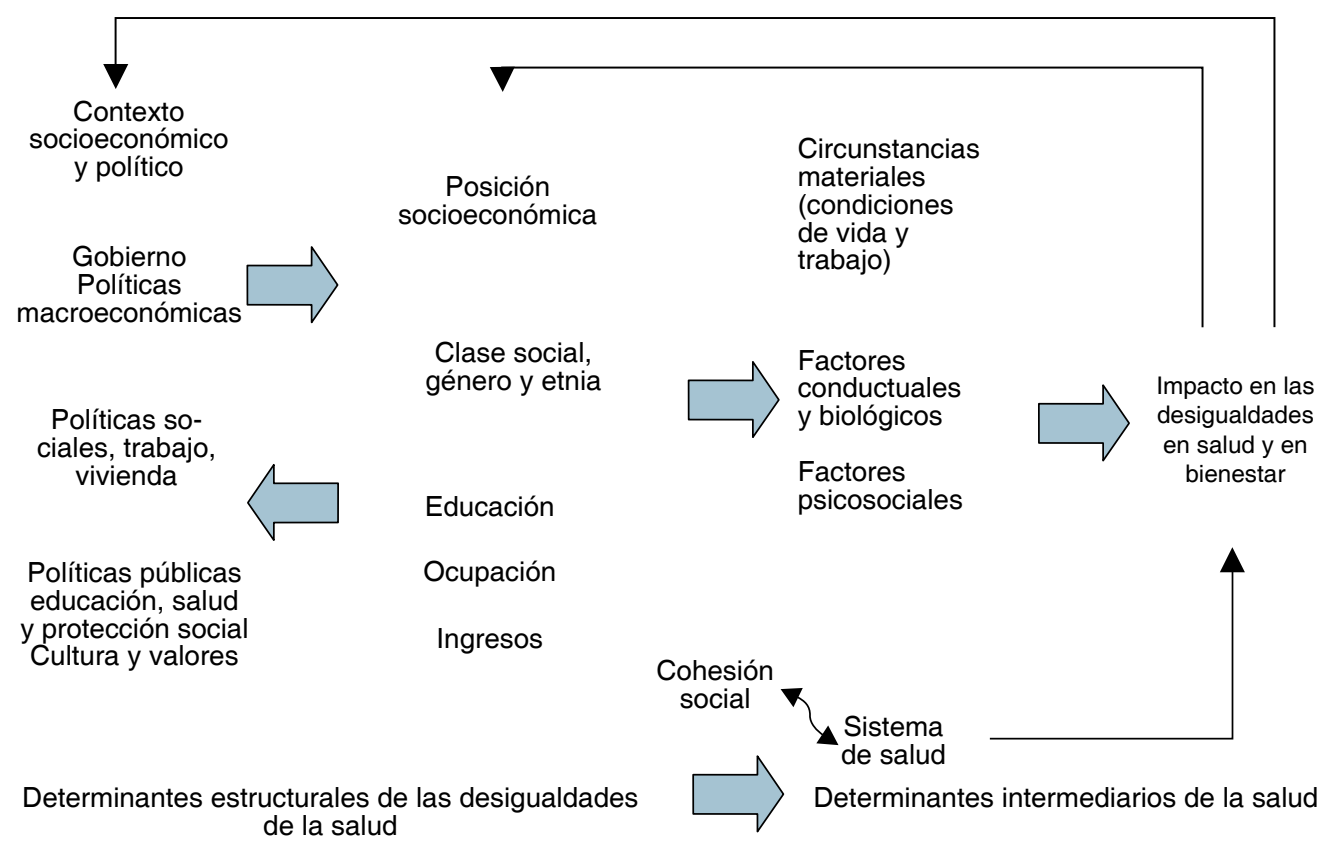

Figura 1. Modelo de determinantes sociales (CDSS-OMS 2007).

llevar a cabo el proceso de análisis con diseño narrativo de vida, según Minayo. ${ }^{11}$

\section{RESULTADOS}

Datos socioeconómicos. Las participantes fueron ocho mujeres con criterios para SM. El rango de edad fue entre 42 y 51 años. Estado civil: solteras, casadas, conviven con familiares y amigos. Escolaridad: sin escolaridad y mayor a nivel técnico. Ocupación: profesora de nivel básico, trabajadoras domésticas y amas de casa. Su nivel socioeconómico es bajo y medio. Sólo dos de ellas mencionan antecedentes familiares para SM, pero la mayoría refiere que sus hermanos padecen SM y la mayoría de los hijos presentan obesidad.

Las participantes viven en una población rural inmersa en la pobreza, que condiciona las oportunidades de trabajo y escolaridad. Se mencionó:

"Nuestro sueldo es bajo y el dinero no alcanza." (Alicia) "No tengo posibilidad de ascenso." (Alma).

"Tengo que trabajar en lo que cae." (Araceli)

Las condiciones de la vivienda se detectaron insalubres; muchos hogares aún carecen de una vivienda individual por familia. Las entrevistadas mencionan:

"Muchos años vivíamos tres familias en una sola casa, una habitación por familia, carecíamos de agua potable, sin drenaje." (Alicia)

La OMS dice que la vivienda debe contar con espacio y servicios necesarios acordes al tamaño de la familia. En lo relacionado con el nivel social, éste se fracciona con mucha facilidad por causa de la pobreza.

Género-trabajo, relaciones familiares, maternidad. Las participantes ingresan un aporte económico con dos o tres jornadas de trabajo; además, realizan las tareas del hogar, la maternidad biológica y la impuesta, en la cual la mujer se hace cargo del cuidado de los padres, hermanos y otros familiares sin momentos de descanso, como es expresado en el siguiente discurso:

"Cocinaba para mi cuñado y mi esposo... Tenía que atender a mis hijas y mi arreglo era rápido... Desayunando en el camino lo que encontrara." (Alicia)

Condiciones de los alimentos. El acceso, creencias y costumbres son puntos claves y cruciales en la población mexicana. Los programas institucionales proveen de información relacionada con la alimentación exclusiva en aquéllos que padecen SM, y las participantes asumen conocer su alimentación apropiada; comentan:

"Pues en la casa se come de una sola dieta, procurando a la familia, pero me han dicho que no debo comer pan, tortillas, refrescos, pasteles... y yo tomo agua, no como tortilla ni pan." (Alicia)

Cuando se dialoga con las personas, mencionan que lo hacen (afirmando/negando), lejos de llevar una alimentación saludable. Lo antes mencionado es parte de su conocimiento, mientras que persisten las costumbres en su forma de alimentación. La entrevista a profundidad nos arroja: 


\begin{tabular}{|c|c|c|c|c|c|}
\hline \multicolumn{2}{|c|}{ Determinantes estructurales } & \multicolumn{4}{|c|}{ Determinantes intermediarios } \\
\hline Economía & Género, Etnia & $\begin{array}{c}\text { Condición } \\
\text { de los alimentos }\end{array}$ & $\begin{array}{l}\text { Condición de } \\
\text { trabajo }\end{array}$ & Biología & Sistema de salud \\
\hline 1. Ingresos & 1. Trabajo & 1. Conocimiento & 1. Situación & 1. Genética & 1. Acceso \\
\hline $\begin{array}{l}\text { 2. Vivienda; falta } \\
\text { de recursos en el } \\
\text { hogar y en la } \\
\text { población }\end{array}$ & $\begin{array}{l}\text { 2. Relaciones } \\
\text { familiares } \\
\text { 3. Maternidad }\end{array}$ & $\begin{array}{l}\text { 2. Acceso } \\
\text { 3. Creencias } \\
\text { 4. Costumbres }\end{array}$ & 2. Sueldo & $\begin{array}{l}\text { 2. Representación } \\
\text { de la salud } \\
\text { 3. Búsqueda de } \\
\text { tratamientos } \\
\text { alternativos }\end{array}$ & $\begin{array}{l}\text { 2. Atención } \\
\text { 3. Continuidad } \\
\text { de la atención }\end{array}$ \\
\hline $\begin{array}{l}\text { Pobreza } \\
\text { Negación } \\
\text { Familias extensas }\end{array}$ & $\begin{array}{l}\text { Cuidar } \\
\text { Tiempo } \\
\text { Atender } \\
\text { Trabajar } \\
\text { Resignación } \\
\text { Tristeza } \\
\text { Trabajo todo el día }\end{array}$ & $\begin{array}{l}\text { Selección } \\
\text { Lo que se puede } \\
\text { Agua } \\
\text { Tortillas } \\
\text { Pan } \\
\text { Refrescos } \\
\text { Frutas }\end{array}$ & $\begin{array}{l}\text { Oportunidad } \\
\text { Expectativas } \\
\text { Lejos } \\
\text { Limitación } \\
\text { Demandante } \\
\text { Poco pago } \\
\text { Calor }\end{array}$ & $\begin{array}{l}\text { Padres } \\
\text { Hermanos } \\
\text { Hijos } \\
\text { Susto } \\
\text { Preocupación } \\
\text { Menopausia } \\
\text { Cansancio } \\
\text { Calambres } \\
\text { Dolor } \\
\text { Hambre } \\
\text { Sed } \\
\text { Bajé de peso } \\
\text { Fui delgada }\end{array}$ & $\begin{array}{l}\text { Atienden } \\
\text { Citas } \\
\text { Médicos } \\
\text { Trato } \\
\text { Lejos } \\
\text { Costo } \\
\text { Plantas medicinales } \\
\text { Licuados } \\
\text { Gotas }\end{array}$ \\
\hline
\end{tabular}

"Como de todo... se me antojan todas las cosas... hay alimentos que no los puedo dejar, me siento débil o con molestias gástricas." (Alicia)

La mayoría carece de una alimentación especial; a ello se suma el alto costo de la vida y los bajos salarios, que facilita el consumo de comida rica en grasas y carbohidratos.

La biología humana incluye la genética como un precursor para salud y/o enfermedad; algunos se encuentran convencidos por herencia, otros dudan de la información, pues su caso muestra diferente comportamiento o bien, no hay aceptación de su problema. Lo expresan en representación de la salud y la búsqueda de tratamientos alternativos:

"Siempre ando con la glucosa alta." (Amanda)

"Mis dos hijas padecen obesidad." (Alicia)

"Siempre porque se me baja el azúcar, he llegado hasta 50." (Ana)

"Acá se acostumbran licuados con nopal, xoconostle... yo sólo con unas gotitas me compuse; siempre los remedios caseros hacen buenos efectos." (Araceli)

Lo atribuyen a que cuidarse no implica gran importancia, resignación y el sentirse mal, y es por el avance de la enfermedad.

Atención y acceso a la salud. México cuenta con programas de cobertura a la población en general. A través de sus vivencias en la atención, ellas, de quienes la mayoría tiene más de 10 años con la enfermedad, mencionan:

"No asisto al médico, tomo una pastillas que me recomendaron, ni me checo" (Ángela)

"Casi no nos atienden, no hay especialista... la asistente me dio cita para seis meses [...] no hay fichas para consulta." (Araceli)

"Hasta que ya tenía mi pie gangrenado y el doctor me dijo que tenía alta mi azúcar." (Ana)

El siguiente comentario difiere:

"Yo me sentía mal y el doctor de mi clínica me revisó y me dijo que no tenía nada y mejor no voy por el mal trato que me dan." (Araceli)

Mediante los informes de las participantes se da la particularidad de autocuidado, las complicaciones, los significados de la salud-enfermedad, la atención, la libertad de recurrir con quien ellas tienen confianza y puedan cubrir el costo de la atención.

\section{DISCUSIÓN}

Los elementos principales son los determinantes sociales en salud en las personas que padecen SM. Retomando la perspectiva de J. Breilh,"El impacto a la salud, la cual no sólo depende de los servicios de salud ni de las características biológicas humanas, sino que a su vez está deter- 
minada por los modos, condiciones y estilos de vida que la sociedad impone a las poblaciones". ${ }^{6}$

La pobreza, como expresión de desigualdad socioeconómica, ${ }^{13}$ y las desigualdades sociales tienen su expresión en las condiciones de salud, casi siempre en perjuicio de los pobres y más pronunciadas con los indicadores de mala salud. ${ }^{14}$

En lo relacionado con el género, las mujeres proveen el cuidado a su familia y la atención a su padecimiento queda en último lugar. ${ }^{15}$ Un estudio por Hamui y colaboradores ${ }^{16}$ habla acerca de cómo las actividades familiares y laborales generan sobrecarga física y emocional, mermando la salud, relacionada con las enfermedades crónicas. Ellas acuden a la medicina alternativa prometedora de alivio y dejan de lado las recomendaciones de los servicios de salud. García ${ }^{9}$ menciona la planificación y gestión de servicios, ofertando servicios accesibles y adecuados de apoyo a las mujeres, en el que se aborden estrategias para eliminar la situación de desigualdad de género en su cuidado.

En relación con la adherencia a la alimentación, los hallazgos de Río y su grupo muestran situaciones similares a las del presente estudio, con dificultad para adaptase a un régimen dietético. ${ }^{17}$ Por otra parte, $27.4 \%$ de la población vive en vulnerabilidad por carencia de acceso a los alimentos. ${ }^{8}$

Sobre los aspectos biológicos, las familias en su mayoría son extensas, por lo cual requieren atención especial, pues de ahí se genera la herencia de las costumbres, los hábitos alimentarios y el cuidado. La familia no está preparada para afrontar este problema, pues en un gran número de ellos el SM no fue adquirido por genética.

Es de notar la atención médica o búsqueda de la curación por medio de la medicina alternativa para disminuir sus dolencias. Por una parte los servicios de salud parecen no garantizar una satisfacción a la población y la capacidad resolutiva de los servicios. ${ }^{5}$

\section{CONCLUSIONES}

A partir de retos, a través de organismos como la OMS y la OPS, y con la implementación de programas nacionales estratégicos orientados hacia las enfermedades crónicas, se ha buscado la participación ciudadana con iniciativas centradas en la promoción a la salud que integren la participación de la población en el cuidado de su salud colectiva. ${ }^{18}$

Los servicios sanitarios deben fortalecer sus competencias técnico-científicas, requeridas en la atención primaria a la salud. Breilh afirma que es importante "dar solución a las necesidades de los sectores más vulnerables de la población, como las personas pobres y marginadas, que enferman más y mueren antes que las personas que gozan de mejor posición social, y son una razón de peso subyacente a las grandes diferencias". ${ }^{6}$

Para finalizar, es indudable que los enfoques dados a la investigación sobre determinantes sociales en salud van atendiendo las esferas física, psicológica, social y econó- mica, desde el aporte y la experiencia de las participantes. Se requiere rescatar la utilidad de estas investigaciones para la toma de decisiones y así realizar acciones más allá de los consultorios y hospitales.

\section{BIBLIOGRAFÍA}

1. Breilh PNJ. Epidemiología entrefuegos. Medellín: Memorias de la Asociación Latinoamericana de Medicina Social; 1994.

2. Castellanos PL. Sobre el concepto de salud-enfermedad. Un punto de vista epidemiológico. Cuadernos Médico-Sociales. Caracas, Venezuela; 1987.

3. González BMA. Investigación en sistemas de salud. Contribuciones selectas del INSP en el XV Aniversario del CISS. Cuernavaca, México; 2011. 11-12.

4. Cabrera A, Camacho I, Cortés N, González R, Ortiz J. ¿Determinación social o determinantes sociales de la salud? Por una discusión acerca de los informes de la CDSS desde la perspectiva de la medicina social latinoamericana. Universidad Autónoma Metropolitana; 2011. 25-29.

5. Secretaría de Salud. Programa Nacional de Salud 20072012.

6. Breilh PNJ. Los determinantes sociales vs. determinación social. Brasil; 2012 (2007-2011). [En línea]. [Consultado en febrero de 2014.] Disponible en: http://www.slideshare.net/ mjbreilh/epidemiologa-critica-jaime-breilh-oct-2011.

7. Comisión de Determinantes Sociales (CDSS-2005-2008) OMS; 2010.

8. Gutiérrez JP, Rivera DJ, Shamah LT, Villalpando HS, Franco A, Cuevas NL et al. Encuesta Nacional de Salud y Nutrición 2012. Resultados Nacionales. Rev INSP México. 2012: 180-185.

9. García GE, De la Llata RM, Kaufe HM, Tusié LMT, Calzada LR, Vázquez VV et al. La obesidad y el síndrome metabólico como problema de salud pública. Revista de Salud Pública Méx. 2008; 50 (6): 530-547.

10. Zimmet $P$, Alberti G, Serrano M. Una nueva definición mundial del síndrome metabólico propuesta por la Federación Internacional de Diabetes: fundamento y resultados. Rev Cardiología. 2005; 58 (12): 1371-1376.

11. Minayo MCS. La artesanía de la investigación cualitativa. Buenos Aires. Lugar editorial, 2009. 125-131.

12. Reglamento de la Ley General de Salud en Materia de Investigación para la Salud [En línea]. [Consultado el 22 de septiembre de 2013]. Disponible en: http://www.salud.gob.mx/ unidades/cdi/nom/compi/rlgsmis.html

13. González A, Simental L, Elizondo S, Sánchez J, Gutiérrez G, Guerrero F. Prevalencia del síndrome metabólico entre adultos mexicanos no diabéticos, usando las definiciones de la OMS, NCEP-ATPIIla e IDF. Rev Médica del Hospital General. 2008; 71 (1): 11-19.

14. Garriga Y, Navarro J, Saumell A, Serviat T, León HJ, García S. Determinantes de la salud: el rol de la inequidad en salud. 2012. [En línea]. [Consultado el 30 de diciembre de 2013] . Disponible en: http://bvs.sld.cu/revistas/infd/n1512/ infd07212.htm

15. García CMM. Cuidados de salud, género y desigualdad. Escuela Andaluza de Salud Pública. Granada [En línea]. [Consultado el 30 de diciembre de 2013] . Disponible en: http://www.pacap.net/es/publicaciones/pdf/comunidad/5/ editorial.pdf

16. Hamui SL, Fuentes GR, Aguirre HR, Ramírez ROF. Estudio de satisfacción de la atención Médica. México: UAM; 2013. 70 . 
Enf Neurol (Mex) • 2014 • Vol. 13 • Núm. 3 • 112-117

17. Rio VJS, Cruz QF, Villaverde GC, Prados PD. Adherencia terapéutica en hipertensos. Rev Index Enferm. 2006; 15 (54).

18. Organización Mundial de la Salud. Obesidad y sobrepeso. 2012; 311 [En línea]. [Consultado el 30 de agosto de 2013]. Disponible en: http://www.who.int/mediacentre/factsheets/fs311/es/
Dirección para Correspondencia:

Abigail Fernández-Sánchez.

4a. privada Narciso Mendoza Núm. 7, Col. Santa

María Ahuacatitlán, Cuernavaca, Morelos, 62100.

E-mail: abifer2002@yahoo.com 